\title{
Scutellarin-treated exosomes increase claudin 5, occludin and ZO1 expression in rat brain microvascular endothelial cells
}

\author{
XIAOQIN ZHONG ${ }^{1-3^{*}}, \mathrm{CHUANJIN} \mathrm{LUO}^{4 *}, \mathrm{MINZHEN} \mathrm{DENG}^{1-3}$ and MIN ZHAO ${ }^{1-3}$ \\ ${ }^{1}$ Department of Neurology, Guangdong Provincial Hospital of Chinese Medicine; ${ }^{2}$ The Second Clinical College, \\ Guangzhou University of Chinese Medicine, Guangzhou, Guangdong 510120; ${ }^{3}$ Department of Neurology, \\ Guangdong Provincial Academy of Chinese Medical Sciences, Guangzhou, Guangdong 510006; ${ }^{4}$ Department of Cardiology, \\ The First Hospital Affiliated to Guangzhou University of Chinese Medicine, Guangzhou, Guangdong 510405, P.R. China
}

Received March 5, 2018; Accepted August 2, 2018

DOI: $10.3892 / \mathrm{etm} .2019 .7562$

\begin{abstract}
Homocysteine has been reported to be an independent risk factor for stroke. Scutellarin (Scu) dilates cerebral blood vessels and promotes anti-platelet aggregation; however, the mechanism by which Scu and Scu-treated exosomes protect against cerebrovascular disease is unclear. The aim of the present study was to investigate the mechanisms underlying the effects of Scu and Scu-treated exosomes on tight junction proteins in the blood-brain barrier. Rat brain microvascular endothelial cells (RBMVECs) were cultured and divided into five groups: Control, model, Scu, exosomes derived from RBMVECs and exosomes derived from RBMVECs after Scu administration. MTT, lactate dehydrogenase (LDH) and nitric oxide (NO) assays were performed to assess cell viability and injury. Reactive oxygen species (ROS) levels were detected using spectrophotometry and immunofluorescence. Western blotting and immunofluorescence were performed to measure cluster of differentiation (CD) 63 , claudin 5, occludin and tight junction protein 1 (ZO1) expression. The results revealed that treatment with Scu and Scu-treated exosomes enhanced cell viability, reduced cell injury, increased NO levels, upregulated CD63, claudin 5, occludin and ZO1, and decreased LDH and ROS levels. These data suggest that Scu and Scu-treated exosomes protect homocysteine-induced RBMVECs via increased claudin 5, occludin and ZO1 expression.
\end{abstract}

Correspondence to: Dr Minzhen Deng or Dr Min Zhao, Department of Neurology, Guangdong Provincial Hospital of Chinese Medicine, 111 Dade Road, Guangzhou, Guangdong 510120, P.R. China

E-mail: dengmz1@126.com

E-mail: cassiesandra@163.com

${ }^{*}$ Contributed equally

Key words: Scutellarin, exosomes, homocysteine, blood-brain barrier, stroke

\section{Introduction}

Stroke is the leading cause of mortality and disability in China and the second most common cause of mortality worldwide $(1,2)$. It has previously been reported that homocysteine (Hcy) is an important risk factor for stroke $(3,4)$. High levels of Hcy are automatically oxidized to produce reactive oxygen species (ROS), which may damage the cardiovascular and immune systems (5). Hcy is recognized as an independent risk factor for cerebrovascular disease, but its role in pathogenesis remains unclear.

The blood-brain barrier (BBB) is the structural basis that maintains the internal environment of the central nervous system (6). BBB destruction is an important pathological characteristic of many neurological diseases (6). Lominadze et al (7) reported that cerebrovascular disease was associated with BBB damage, while Kamath et al (8) reported that high Hcy concentrations inhibit tight junction (TJ) protein expression in cerebrovascular endothelial cells, which results in damage to the TJ structure. Lee's study revealed that high Hcy levels damaged the BBB structure and increased BBB permeability (9). Tyagi et al (10) suggested that Hcy activates matrix metalloproteinases (MMPs), whose overexpression reduces the ability of cells to connect and alters the basement membrane, resulting in destruction of the blood-brain barrier.

Scutellarin $(\mathrm{Scu})$ is an active component of Erigerontis Herba, which exhibits anti-inflammatory, antioxidative and protective effect on endothelial cells (11). Erigerontis Herba has been widely used in the treatment of cardiovascular and cerebrovascular diseases (12). Yuan et al (12) reported that Scu inhibited microglial activation and alleviated the symptoms of neuroinflammation, which contributed to the clinical treatment of cerebral ischemia. Du et al (13) demonstrated that Scu activated the endothelial cGMP activated protein kinase G (PKG) pathway to protect cerebral vascular endothelial cells. Furthermore, Scu has been revealed to prevent learning and memory defects in a rat model of Alzheimer's disease via reducing oxidative stress and inflammation (14).

Exosomes are cell membrane-derived vesicles that influence cellular activity by transporting proteins, lipids and nucleic acids to target cells (15). It has been reported that exosomes are able to promote functional recovery and neurovascular plasticity following stroke (16), as well as serving 
an important role in the pathogenesis of Alzheimer's disease, Parkinson's disease, prion disease and other neurological diseases (17). Exosomes may therefore have potential as clinical treatments for central nervous system diseases. The aim of the present study was to investigate the expression of TJ proteins claudin 5, occludin and TJ protein 1 (ZO1) in the BBB using Hcy-induced rat brain microvascular endothelial cells (RBMVECs).

\section{Materials and methods}

Cell culture. RBMVECs (Saiqi Biological Engineering Co., Ltd., Shanghai, China) were seeded in $25-\mathrm{cm}^{2}$ polystyrene flasks (Corning Incorporated, Corning, NY, USA) with $4.5 \mathrm{~g} / \mathrm{ml}$ glucose Dulbecco's modified Eagle's medium (DMEM) supplemented with $10 \%$ heat-inactivated fetal bovine serum (both Gibco; Thermo Fisher Scientific, Inc., Waltham, MA, USA). The cells were incubated at $37^{\circ} \mathrm{C}$ in an atmosphere containing $5 \% \mathrm{CO}_{2}$. DMEM was replaced every $48 \mathrm{~h}$.

Exosome extraction. Exosomes derived from normal RBMVECs following Scu administration (SE) and exosomes derived from control RBMVECs (CE) were extracted from cells and incubated with $100 \mu \mathrm{mol} / 1 \mathrm{Scu}$ or DMEM for $24 \mathrm{~h}$, respectably. A total of $5 \mathrm{ml}$ supernatant was collected from each flask and centrifuged at $3,000 \mathrm{x}$ g for $15 \mathrm{~min}$ at room temperature to exclude cell debris. The supernatant was transferred to a new sterile centrifuge tube and the $1 \mathrm{ml}$ ExoQuick-TC (Tissue Culture Media Exosome Precipitation Solution; cat. no. 170306-001; System Biosciences, Palo Alto, CA, USA) was added. The solution was vortexed overnight at $4^{\circ} \mathrm{C}$. The suspension was then centrifuged at room temperature at $1,500 \mathrm{x}$ g for $30 \mathrm{~min}$. Exosome-containing pellets were resuspended in $500 \mu 1 \mathrm{PBS}$ for intervention experiments.

Cell viability. Cells were seeded at a density of $1 \times 10^{4}$ cells/well in 96-well culture plates and incubated for 24 or $48 \mathrm{~h}$ at $37^{\circ} \mathrm{C}$ in an atmosphere containing $5 \% \mathrm{CO}_{2}$. Cells were incubated with aggregated intervention medications. A total of $10 \mu \mathrm{l}$ MTT $(5 \mathrm{mg} / \mathrm{ml}$; Sigma-Aldrich; Merck KGaA, Darmstadt, Germany) was added to each well and cells were incubated at $37^{\circ} \mathrm{C}$ for $4 \mathrm{~h}$. The culture medium was discarded and $150 \mu$ l of dimethyl sulfoxide (DMSO; Sigma-Aldrich; Merck $\mathrm{KGaA}$ ) was added to dissolve the formazan crystals. The number of viable cells in each well was measured at $490 \mathrm{~nm}$ using a microplate reader (Multiskan Mk3; Thermo Fisher Scientific, Inc.).

Experimental design. After the concentration optimization experiments by MTT assay, RBMVECs were divided into the following groups: Control, model, $\mathrm{Scu}$, exosomes derived from CEs and exosomes derived from normal RBMVECs after SE. The cultured cells in all groups were pretreated with Scu ( $100 \mu \mathrm{mol} / \mathrm{l}), \mathrm{CE}$ (40\% suspension of control exosomes in $1 \mathrm{ml}$ of DMEM) and SE (40\% suspension of Scu exosomes in of $1 \mathrm{ml}$ DMEM) for $30 \mathrm{~min}$, followed by Hcy exposure $(2.5 \mathrm{mmol} / \mathrm{l}$; cat. no. Z20J8H28854; Shanghai Yuanye Bio-Technology Co., Ltd, China) for $48 \mathrm{~h}$.
Measurement of lactate dehydrogenase (LDH) and nitric oxide $(N O)$. $\mathrm{LDH}$ and $\mathrm{NO}$ release into the culture medium from dead cells was assessed using an LDH cytotoxicity assay kit (cat. no. 20170314) and an NO detection kit (cat. no. 20170407; both Nanjing JianCheng Bioengineering Institute, Nanjing, China). Cells were lysed with $1 \%$ SDS and centrifuged at $4{ }^{\circ} \mathrm{C}$, $2,000 \mathrm{x} \mathrm{g}$ for $10 \mathrm{~min}$. The supernatants from each group were assessed according to the manufacturer's protocol.

ROS detection. Intracellular ROS levels were monitored using 2',7'-dichlorofluorescein diacetate (DCFH-DA; cat. no. 170V; Beijing Vigorous Biotechnology Co., Ltd, China) to identify the role of ROS in Scu and Scu-treated exosomes. RBMVECs were seeded in 24 well plates at a density of $2 \times 10^{5}$ cells/well and incubated overnight at $37^{\circ} \mathrm{C}$. Cells were subsequently incubated with or without intervention drugs (Scu, CE and SE) for $48 \mathrm{~h}$. The cells were then treated with DCFH-DA $(5 \mu \mathrm{M})$ at $37^{\circ} \mathrm{C}$ for $90 \mathrm{~min}$. Fluorescence microscopy (magnification, x400; U-SPT; Olympus Corporation, Tokyo, Japan) was used to measure the fluorescence intensity of the treated cells.

RBMVECs $\left(1 \times 10^{4}\right.$ in $\left.200 \mu \mathrm{l}\right)$ were seeded in quadruplicate in a 96-well plate and incubated with $2.5 \mathrm{mmol} / \mathrm{l} \mathrm{Hcy}$ for $48 \mathrm{~h}$ in the presence or absence of intervention drugs (Scu, CE and SE). The cultured cells were washed three times with PBS (pH 7.4) and incubated in DMEM containing DCFH-DA $(5 \mu \mathrm{M})$ at $37^{\circ} \mathrm{C}$ for $90 \mathrm{~min}$. The cells were washed with prewarmed PBS and covered with $100 \mu \mathrm{l}$ of DMEM. The fluorescence intensity (FI) of each well was measured using a microplate reader at $485 / 530 \mathrm{~nm}$.

Detection of cluster of differentiation (CD)63, claudin-5, ZO1 and occludin by immunofluorescence. Cells were incubated with $2.5 \mathrm{mmol} / \mathrm{l} \mathrm{Hcy}$ for $48 \mathrm{~h}$ in the presence or absence of intervention drugs (Scu, CE and SE). Briefly, cells were grown on cover slips, and $4 \%$ paraformaldehyde was used to fix the cells for $30 \mathrm{~min}$ at room temperature. Cells were subsequently blocked in $10 \%$ goat serum (Gibco; Thermo Fisher Scientific, Inc.) for $20 \mathrm{~min}$ at $37^{\circ} \mathrm{C}$. Cells were treated with antibodies against CD63 (cat. no. o67346b; OmnimAbs, Alhambra, CA, USA), claudin-5 (cat. no. 6679g62), ZO1 (cat. no. 3268f94; both Affinity Biosciences, USA) and occludin (cat. no. o29813c; OmnimAbs) for $1 \mathrm{~h}$ at $37^{\circ} \mathrm{C}$ (1:50 dilution). Cells were subsequently incubated for $1 \mathrm{~h}$ at $37^{\circ} \mathrm{C}$ with corresponding secondary antibodies conjugated to FITC. Cells were subsequently washed three times with PBS and stained with DAPI $(1 \mu \mathrm{g} / \mathrm{ml}$ in PBS $)$ at $37^{\circ} \mathrm{C}$ in the dark. The coverslips were mounted and cells were observed under a light microscope (U-SPT; Olympus Corp., Tokyo, Japan). Data were analyzed by using Medical Image Analysis Software 16.0 (MIAS, Warrendale, WA, USA).

Western blotting. RBMVECs were harvested and lysed using phenylmethane sulfonyl fluoride-containing lysis buffer (Beyotime Institute of Biotechnology, Haimen, China). Proteins were quantified using a BCA protein assay kit (Beyotime Institute of Biotechnology). Equal amounts of protein $(30 \mu \mathrm{g})$ were separated by $12 \%$ SDS-PAGE and transferred onto a polyvinylidene fluoride membrane. The membrane was blocked with 5\% nonfat skim milk in TBST for $1 \mathrm{~h}$ at room temperature followed by incubation with primary antibodies against CD63, claudin-5, ZO1, occludin and GAPDH (cat. no. 10; Cell 

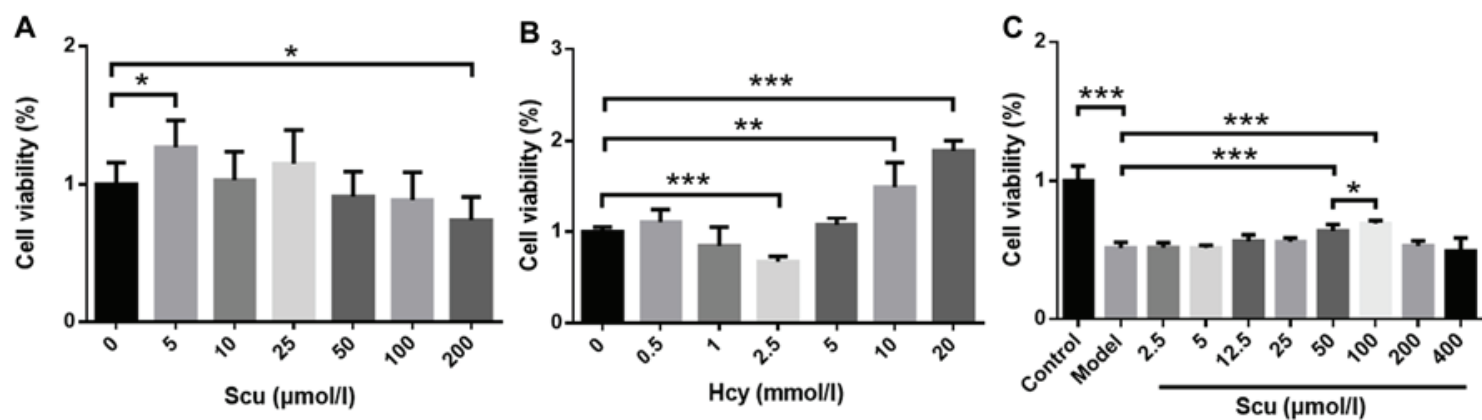

Figure 1.Effects of Scu and Hcy on RBMVEC viability. RBMVEC viability following exposure to (A) $200 \mu \mathrm{mol} / 1 \mathrm{Scu}$ or (B) $2.5 \mathrm{mmol} / 1 \mathrm{Hcy}$ for $24 \mathrm{~h}$. (C) The viability of Hcy-induced RBMVECs following treatment with 50-100 $\mu$ mol/1 Scu for $24 \mathrm{~h}$. (A) ${ }^{*} \mathrm{P}<0.05 \mathrm{vs} .0 \mu \mathrm{mol} / 1$ Scu group; (B) ${ }^{* * *} \mathrm{P}<0.01$ and ${ }^{* * * *} \mathrm{P}<0.001 \mathrm{vs} .0 \mathrm{mmol} / 1 \mathrm{Hcy}$ group; (C) ${ }^{*} \mathrm{P}<0.05,{ }^{* *} \mathrm{P}<0.01$ and ${ }^{* * *} \mathrm{P}<0.001$ vs. Model group and ${ }^{*} \mathrm{P}<0.05$ with a bar linking the $50 \mu \mathrm{mol} / 1$ Scu group and $100 \mu$ mol/1 Scu group with an asterisk. $\mathrm{n}=6$. Scu, Scutellarin; Hcy, homocysteine; RBMVEC, rat brain microvascular endothelial cell; Model, $2.5 \mathrm{mmol} / 1$ Hcy-induced RBMVECs.
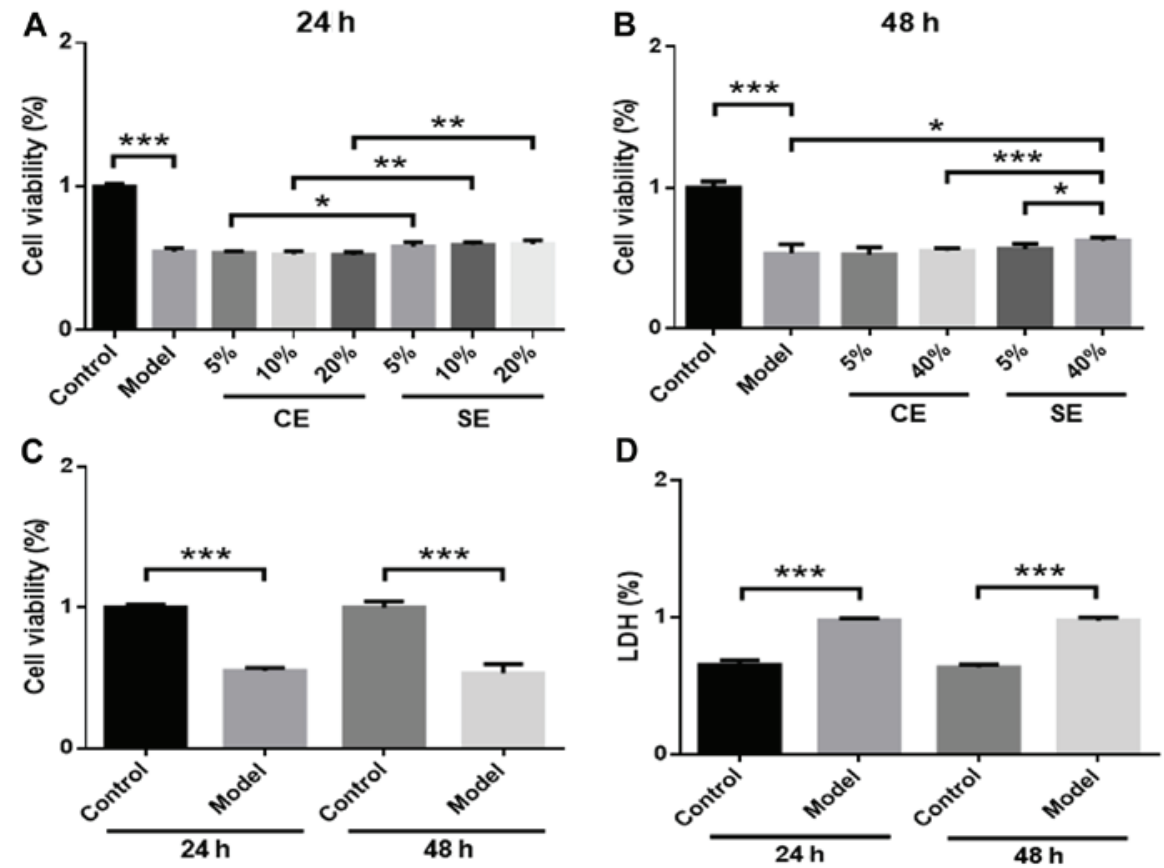

Figure 2. Effects of pretreated exosomes on Hcy-induced RBMVEC viability. Cells were treated with 5-20\% CE or SE for (A) $24 \mathrm{~h}$ or (B) $48 \mathrm{~h}$. (C) Cell viability and (D) LDH expression in the model and control groups was assessed at 24 and $48 \mathrm{~h} .{ }^{*} \mathrm{P}<0.05,{ }^{* *} \mathrm{P}<0.01$ and ${ }^{* * * *} \mathrm{P}<0.001$ with a bar linking the groups with an asterisk. n=6. Hcy, homocysteine; RBMVEC, Rat brain microvascular endothelial cell; LDH, lactate dehydrogenase; CE, control-treated exosomes; $\mathrm{SE}$, Scutellarin-treated exosomes; Model, $2.5 \mathrm{mmol} / \mathrm{l} \mathrm{Hcy-induced} \mathrm{rat} \mathrm{brain} \mathrm{microvascular} \mathrm{endothelial} \mathrm{cells.}$

Signaling Technology, Inc., Danvers, MA, USA) overnight at $4^{\circ} \mathrm{C}$ (1:1,000 dilution). The membrane was washed with TBST and incubated with horseradish peroxidase conjugated secondary antibodies for $1 \mathrm{~h}$ at $37^{\circ} \mathrm{C}$ (1:2,000 dilution; cat/no. 7074s; Cell Signaling Technology, Inc.). Proteins were detected using an ECL detection kit and Image-Lab software-5.2.1 (both Bio-Rad Laboratories, Inc., Hercules, CA, USA).

Statistical analysis. Data are expressed as the mean \pm standard deviation. Significant differences between groups were determined using one-way analysis of variance followed by Bonferroni's post hoc test for multiple comparisons. Correlations between LDH, NO, ROS, CD63, claudin 5, occludin and ZO1 expression were identified using Pearson's correlation analysis. All data analyses were performed using SPSS 16.0 statistical software (SPSS, Inc., Chicago, IL, USA). P $<0.05$ was considered to indicate a statistically significant difference.

\section{Results}

Effect of Scu on Hcy-induced RBMVEC viability. Cell viability increased in the presence of $5 \mu \mathrm{mol} / 1 \mathrm{Scu}(\mathrm{P}<0.05)$ (Fig. 1A) and decreased in the presence of $200 \mu \mathrm{mol} / 1 \mathrm{Scu}$ $(\mathrm{P}<0.05)$ (Fig. 1A) compared with untreated RBMVECs. Treatment with $2.5 \mathrm{mmol} / \mathrm{l}$ Hcy significantly decreased the viability of RBMVECs compared with the control $(\mathrm{P}<0.001$; Fig. 1B), while viability was increased following treatment 10-20 mmol/1 Hcy $(\mathrm{P}<0.001)$ (Fig. 1B). The effects of 2.5-400 $\mu \mathrm{mol} / 1 \mathrm{Scu}$ on the activity of RBMVECs induced with $2.5 \mathrm{mmol} / 1 \mathrm{Hcy}$ were then investigated. Cell viability increased significantly in the presence of 50 or $100 \mu \mathrm{mol} / 1$ Scu compared with the model group $(\mathrm{P}<0.001)$ (Fig. 1C). Furthermore, $100 \mu \mathrm{mol} / \mathrm{l}$ Scu-treated cell viability was significantly higher compared with $50 \mu \mathrm{mol} / 1 \mathrm{Scu}$-treated cells $(\mathrm{P}<0.05)$ (Fig. 1C). 
A

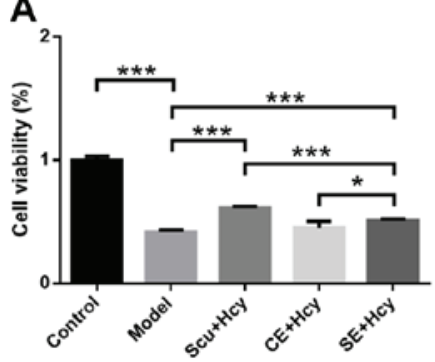

B

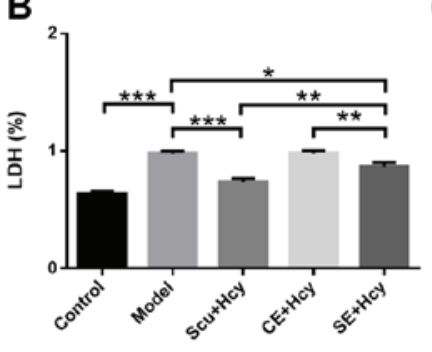

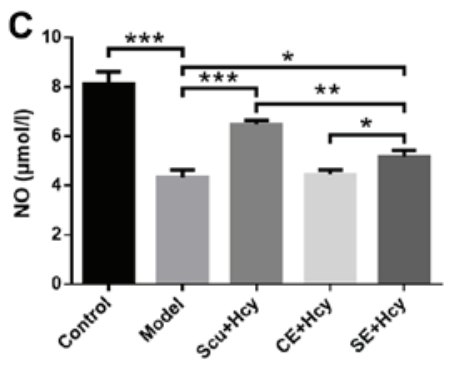

Figure 3. Effects of pretreated exosomes on LDH and NO levels. (A) Cell viability, (B) LDH expression and (C) NO levels in the Scu and SE groups. * $<0.05$, ${ }^{* *} \mathrm{P}<0.01$ and ${ }^{* * *} \mathrm{P}<0.001$ with a bar linking the groups with an asteris. $\mathrm{n}=6$. LDH, lactate dehydrogenase; NO, nitric oxide; Scu, Scutellarin; SE, Scu-treated exosomes; CE, control treated exosomes; Model, $2.5 \mathrm{mmol} / 1$ homocysteine-induced rat brain microvascular endothelial cells.

A

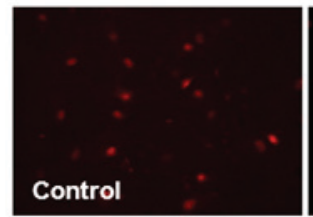

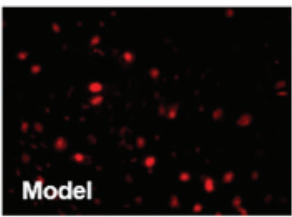

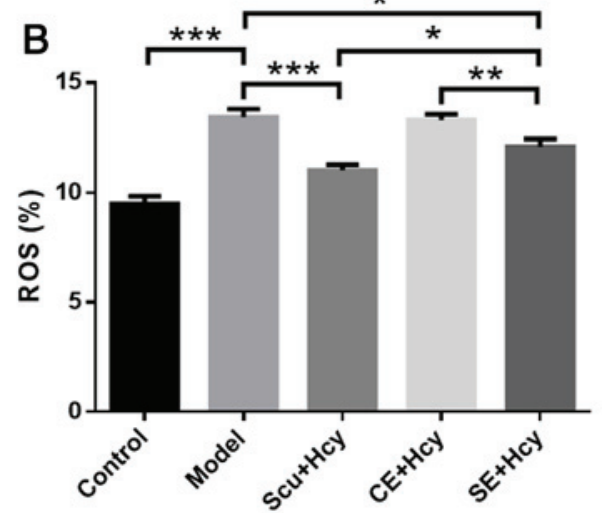

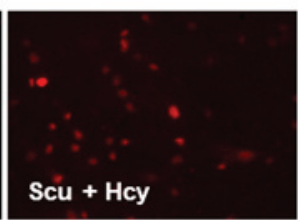
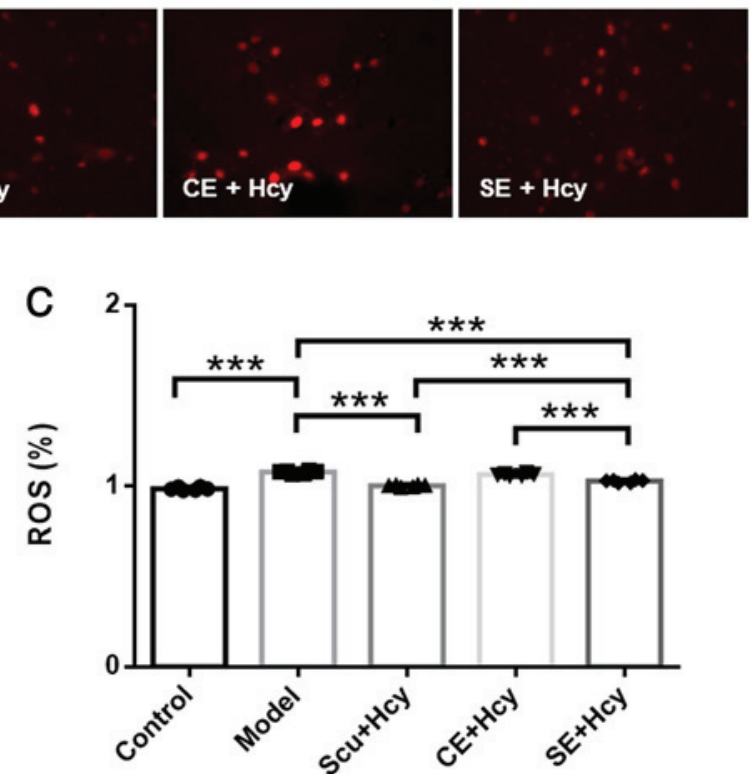

Figure 4. Effect of Scu and Hcy on ROS levels. (A) Representative fluorescence microscopy images of rat brain microvascular endothelial cells (magnification, $\mathrm{x} 400 ; \mathrm{n}=3$ ). (B) Quantified results of ROS fluorescence microscopy. ROS expression was detected using fluorescence microscopy. (C) ROS expression as detected using a fluorescence microplate reader. ${ }^{*} \mathrm{P}<0.05,{ }^{* *} \mathrm{P}<0.01$ and ${ }^{* * *} \mathrm{P}<0.001$ with a bar linking the groups with an asteris. $\mathrm{n}=6$. Scu, Scutellarin; Hcy, homocysteine; ROS, reactive oxygen species; Model, $2.5 \mathrm{mmol} / 1 \mathrm{Hcy}$-induced rat brain microvascular endothelial cells; CE, control-treated exosomes; SE, Scu-treated exosomes.

Effects of pretreated exosomes on Hcy-induced RBMVEC viability. RBMVECs were incubated with different amounts of exosomes $(5,10,20$ or $40 \%$ in DMEM) in the presence of $2.5 \mathrm{mmol} / \mathrm{l} \mathrm{Hcy}$ for 24 or $48 \mathrm{~h}$. No significant changes in cell viability were observed in the 5,10 or $20 \%$ CE and SE groups compared with the model group (Fig. 2A). However, cell viability was significantly higher in the SE group compared with the CE group at $24 \mathrm{~h}(\mathrm{P}<0.05)$ (Fig. 2A). In the $40 \% \mathrm{SE}$ group, cell viability was significantly increased compared with the model group $(\mathrm{P}<0.05)$ (Fig. 2B) and the $40 \%$ CE group at $48 \mathrm{~h}(\mathrm{P}<0.001)$ (Fig. 2B). A dose-effect relationship was observed in the SE group, as treatment with $40 \%$ solution produced increased cell viability significantly more than the $5 \%$ solution $(\mathrm{P}<0.05)$ (Fig. 2B). No significant differences in cell viability or LDH levels were observed at 24 or $48 \mathrm{~h}$, which suggests that the effect of Hcy in the model group was not time-dependent (Fig. 2C and D).

Effect of Scu and Scu-treated exosomes on cell viability, LDH expression and NO levels. RBMVECs were incubated with $2.5 \mathrm{mmol} / \mathrm{l} \mathrm{Hcy}$ for $48 \mathrm{~h}$ along with Scu and Scu-treated exosomes. Cell viability and NO levels in the Scu and SE group were significantly increased $(\mathrm{P}<0.05)$ (Fig. 3A) compared with the model group, while LDH levels were significantly decreased $(\mathrm{P}<0.05)$ (Fig. 3B). In addition, cell viability and $\mathrm{NO}$ levels were significantly higher in the $\mathrm{Scu}$ group compared with the SE group $(\mathrm{P}<0.01)$ (Fig. 3A and C), while LDH levels were significantly lower in the Scu group compared with the SE group $(\mathrm{P}<0.01)$ (Fig. 3B). Cell viability and NO levels were significantly increased $(\mathrm{P}<0.01)$ (Fig. 3A and $\mathrm{C})$ and $\mathrm{LDH}$ levels were significantly decreased $(\mathrm{P}<0.05$; Fig. 3B) in the SE group compared with the CE group.

Effect of Scu and Scu-treated exosomes on ROS. Cells were treated as described above, and ROS levels were detected using fluorescence and spectrophotometry. ROS levels were significantly decreased in the Scu and SE groups compared with the model group $(\mathrm{P}<0.05)$ (Fig. 4). ROS levels were reduced in the Scu and SE groups compared with the CE groups $(\mathrm{P}<0.01)$ (Fig. 4). 

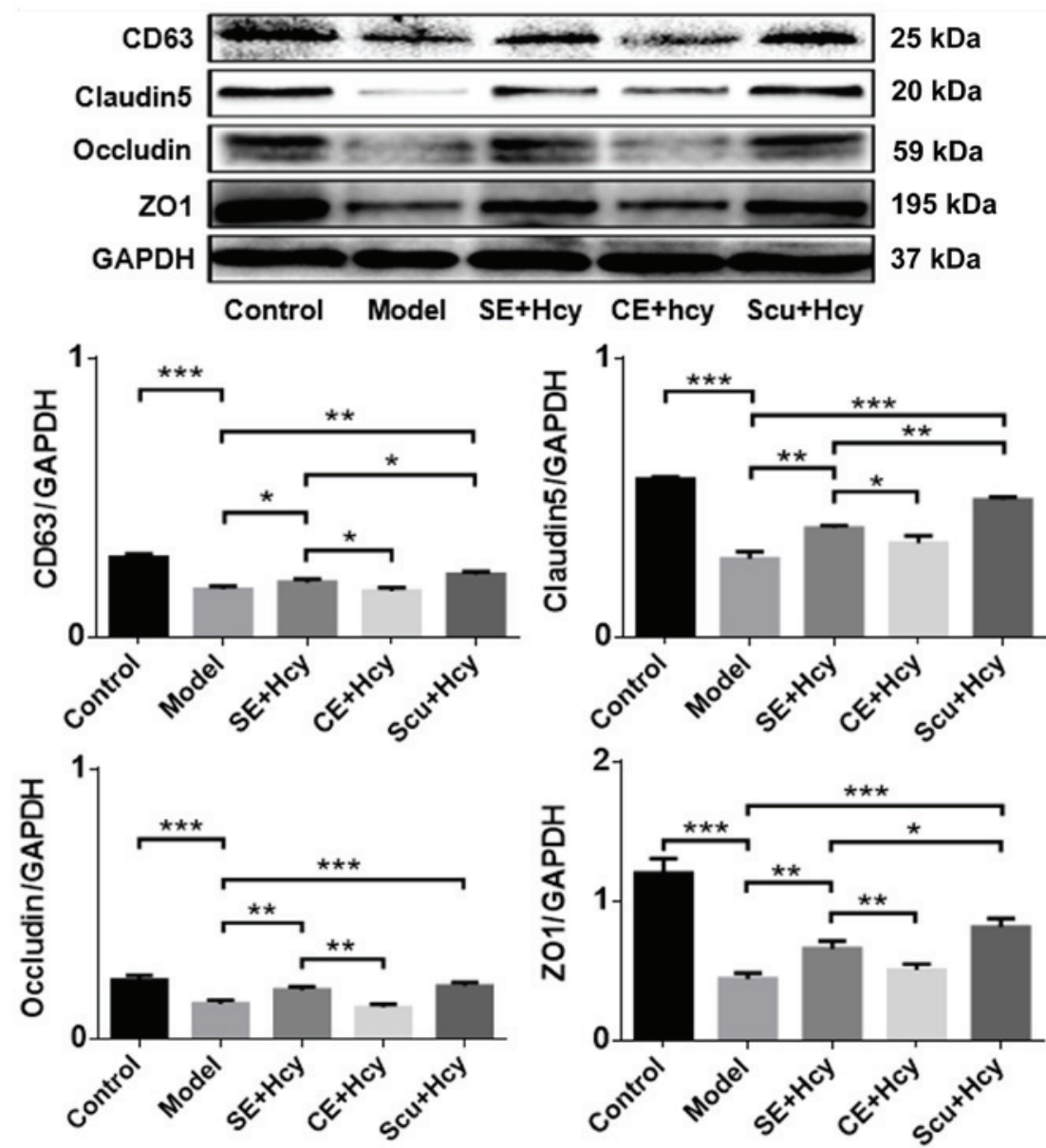

Figure 5. Representative western blot and quantified results for CD63, claudin-5, occludin and ZO1 expression. ${ }^{*} \mathrm{P}<0.05,{ }^{* * *} \mathrm{P}<0.01$ and ${ }^{* * * *} \mathrm{P}<0.001$ with a bar linking the groups with an asterisk. $\mathrm{n}=3$. CD, cluster of differentiation; ZO1, tight junction protein 1; Model, $2.5 \mathrm{mmol} / 1$ Hcy-induced rat brain microvascular endothelial cells; CE, control-treated exosomes; Scu, Scutellarin; SE, Scu-treated exosomes; Hcy, homocysteine.
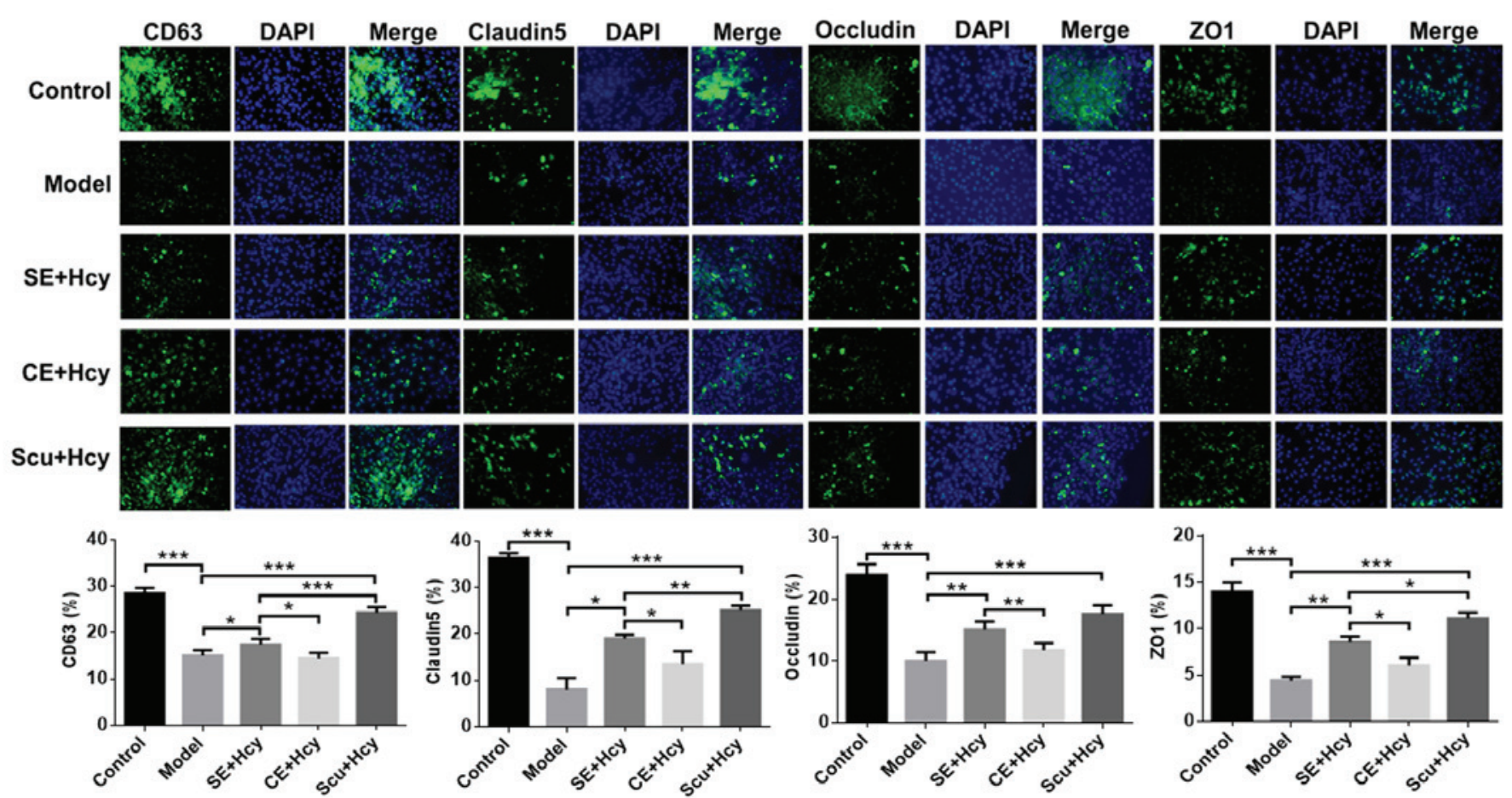

Figure 6. Immunofluorescence of CD63, claudin-5, occludin and ZO1 expression. Magnification, $x 400 ; n=3 .{ }^{*} \mathrm{P}<0.05,{ }^{* * *} \mathrm{P}<0.01$ and ${ }^{* * * *} \mathrm{P}<0.001$ with a bar linking the groups with an asteris. CD, cluster of differentiation; ZO1, tight junction protein 1; Scu, Scutellarin; SE, Scu-treated exosomes; Hcy, homocysteine; CE, control-treated exosomes; Model, $2.5 \mathrm{mmol} / 1$ Hcy-induced rat brain microvascular endothelial cells. 
Table I. Correlation analysis.

\begin{tabular}{|c|c|c|c|c|c|c|c|}
\hline & LDH & NO & ROS & CD63 & Claudin-5 & Occludin & $\mathrm{ZO} 1$ \\
\hline LDH & 1.000 & & & & & & \\
\hline NO & $-0.891^{\mathrm{a}}$ & 1.000 & & & & & \\
\hline ROS & 0.277 & $-0.889^{\mathrm{a}}$ & 1.000 & & & & \\
\hline CD63 & $-0.721^{a}$ & -0.327 & $-0.866^{\mathrm{a}}$ & 1.000 & & & \\
\hline Claudin-5 & $-0.721^{\mathrm{a}}$ & -0.327 & $-0.866^{\mathrm{a}}$ & $0.999^{b}$ & 1.000 & & \\
\hline Occludin & $-0.721^{\mathrm{a}}$ & -0.327 & $-0.866^{\mathrm{a}}$ & $0.999^{\mathrm{b}}$ & $1.000^{\mathrm{b}}$ & 1.000 & \\
\hline $\mathrm{ZO} 1$ & $-0.721^{\mathrm{a}}$ & -0.327 & $-0.866^{\mathrm{a}}$ & $0.999^{\mathrm{b}}$ & $1.000^{\mathrm{b}}$ & $1.000^{\mathrm{b}}$ & 1.000 \\
\hline
\end{tabular}

Effect of Scu and Scu-treated exosomes on CD63, claudin-5, occludin and ZOI expression. The detailed mechanisms underlying the effect of Scu and Scu-treated exosomes were investigated. CD63, claudin-5, occludin and ZO1 expression was significantly increased in the Scu and SE groups compared with the model group $(\mathrm{P}<0.05)$ (Figs. 5 and 6$)$. CD63, claudin-5, occludin and ZO1 expression was significantly increased in the Scu group compared with the SE group $(\mathrm{P}<0.05)$ (Figs. 5 and 6). CD63, claudin-5, occludin and ZO1 expression was significantly higher in the SE group compared with the $\mathrm{CE}$ group $(\mathrm{P}<0.05)$ (Figs. 5 and 6$)$.

Correlations between experimental indicators. The association of LDH levels with NO, ROS, CD63, claudin-5, occludin and ZO1 expression was assessed using Pearson's correlation analysis. The results indicated that LDH expression was significantly negatively correlated with NO, CD63, claudin-5, occludin and ZO1 levels $(\mathrm{P}<0.05)$ (Table I). Furthermore, NO levels were significantly negatively correlated with ROS expression $(\mathrm{P}<0.05)$ (Table I), while ROS expression was significantly negatively correlated with CD63, claudin-5, occludin and ZO1 levels $(\mathrm{P}<0.05)$ (Table I). Significant positive correlations were observed between CD63 and claudin-5, CD63 and occludin, and CD63 and ZO1 expression (all P<0.01) (Table I). Claudin-5 expression was significantly positively correlated with occludin and ZO1 levels $(\mathrm{P}<0.01)$ (Table I).

\section{Discussion}

High Hcy levels induce neuronal apoptosis and BBB destruction via peroxidation, which eventually leads to stroke (18). A recent study demonstrated that TJs close the gap between cerebral vascular endothelial cells, are involved in the blood-brain barrier, transmit intracellular signals and maintain cell growth and differentiation, thereby protecting against neurological diseases (19).

Exosomes are an important intercellular mediator in post-stroke nerve repair and injury, with beneficial effects for patient recovery (20). Scu increases blood flow, dilates blood vessels and lowers blood prophylaxis (12). However, whether high Hcy levels damage the structure and function of the BBB via TJ destruction has not previously been reported. The aim of the present study was to clarify the protective mechanisms of Scu and Scu-treated exosomes on Hcy-induced RBMVECs.

The effects of Scu and Scu-treated exosomes on Hcy-induced RBMVECs were investigated via assessing the biological activity of cells following treatment with Hcy at different concentrations and for different intervals. An LDH detection kit was used to measure changes in LDH release rate. Oxidative stress-related injury and apoptosis increase cell membrane permeability, resulting in the release of LDH (21). As such, LDH activity in the cell culture medium is proportional to cell death (22). For normal RBMVECs, $5-200 \mu \mathrm{mol} / 1 \mathrm{Scu}$ was not observed to cause significant toxicity at $24 \mathrm{~h}$. However, $2.5 \mathrm{mmol} / \mathrm{l} \mathrm{Hcy}$ was observed to be toxic to normal RBMVECs, and so this concentration was selected for subsequent experiments. It was also demonstrated that 50-100 $\mu \mathrm{mol} / 1 \mathrm{Scu}$ protected RBMVECs against Hcy-induced toxicity. Normal exosomes and Scu-treated exosomes $(0-20 \%)$ produced no significant effects on $2.5 \mathrm{mmol} / 1 \mathrm{Hcy}$-induced RBMVECs after $24 \mathrm{~h}$. However, $40 \%$ Scu-treated exosomes had a significant protective effect on cells in the model group after $48 \mathrm{~h}$. Scu-treated exosomes were collected from the cell culture medium following treatment, and the exosomes concentration likely did not reach a maximum. Therefore, a higher concentration $(40 \%)$ of Scu-treated exosomes and a longer exposure duration (48 h) were required to produce significant pharmacological effects. The effects of Hcy on cell viability at different time points (24 and $48 \mathrm{~h}$ ) were investigated using MTT and LDH assays. The results indicated that Hcy-induced damage was not time-dependent in RBMVECs. As such, a treatment duration of $48 \mathrm{~h}$ was selected to ensure consistency across groups. Scu and Scu-treated exosomes reduced Hcy-induced cell damage in the model group, with a greater efficacy observed in the Scu group compared with the SE group in four cell injury measurements.

Hcy produces ROS via self-oxidation, which causes peroxidation of the cell membrane. $\mathrm{O}^{2-}$ formed through the oxidation process combines with $\mathrm{NO}$ and reduces the bioavailability of NO (23). ROS also oxidizes NOS and reduces its NO production capacity (24). Changes in endothelial NO biological activity may be an important marker of vascular endothelial damage (25). NO levels were significantly decreased in the model group, which suggests that Hcy decreased NO activity 
via superoxide activation and induced cerebral vascular endothelial injury. NO levels in the Scu and SE groups were significantly higher compared with the group, which indicated that Scu and Scu-treated exosomes exerted protective effects on cells in the model group. However, the efficacy of Scu was greater than that of Scu-treated exosomes. ROS levels were increased in the model group, confirming that Hcy causes cell damage. ROS levels in the Scu and SE groups were significantly lower compared with the model group, suggesting that RBMVECs in the Scu and SE groups suffered less Hcy-induced damage.

Exosome-mediated communication between cells is achieved via the transfer of biomolecules from a source cell to a target cell (26). CD63 is a lysosomal membrane glycoprotein that is a surface marker protein for exosomes (27). In the present study, CD63 expression was increased in the SE group compared with the Scu group, which in turn was higher compared with the CE group These results suggest that exosomes were enriched in the SE group and provided a certain resistance to the toxic effects of Hcy. These results suggest that $\mathrm{Scu}$ is able to stimulate cells in the model group to secrete exosomes. However, this experiment primarily measured the pharmacological effects of Scu.

It has previously been demonstrated that Scu and Scu-treated exosomes exhibit protective effects on Hcy-induced RBMVECs, suggesting that their protective mechanism reduces Hcy-mediated damage to TJ proteins in the BBB. ZO1 is located in the subcapsular part of the TJ of epithelial cells, which are interconnected via a variety of proteins (28). Occludin and claudin form the primary chain of the linear TJ structure (29). Occludin is directly involved in TJ formation in brain microvascular endothelial cells and its expression is associated with the degree of epithelial cell closure (30). Claudin serves a role in the formation of TJs, but is not specifically expressed in TJs (31). In the present study, claudin-5, occludin and ZO1 expression was significantly reduced in the model group, suggesting that Hcy damages TJ proteins in the BBB. Claudin-5, occludin and ZO1 expression increased significantly following the administration of Scu and its exosomes, with greater efficacy in the Scu group compared with the SE group. These results suggest that $\mathrm{Scu}$ and its exosomes protect cells by increasing the expression of $\mathrm{TJ}$ proteins in the BBB.

Correlation analyses were performed to clarify the associations between experimental indicators. $\mathrm{LDH}$ levels were significantly and negatively correlated with NO levels, which suggests that cell damage was associated with oxidative stress. LDH expression was also significantly and negatively correlated with claudin-5, occludin and ZO1 expression, indicating that cell injury and TJ proteins are closely related. ROS levels were negatively correlated with CD63 expression, which suggests that oxidative stress was associated with decreased lysosomal glycoprotein levels. Finally, CD63 expression was significantly and positively correlated with claudin-5, occludin and ZO1 expression. Together, these results suggest that an increase in lysosomal glycoprotein levels or in exosomes is associated with the integrity of TJ proteins.

In conclusion, Scu and Scu-treated exosomes increased NO levels as well as CD63, claudin-5, occludin and ZO1 expression in Hcy-induced RBMVECs, while LDH and ROS levels were reduced. Together, these effects reduce cell damage and protect the structure and function of the BBB. However, the present study was limited by the lack of accurate identification of exosomes and the lack of in-depth exosome study.

\section{Acknowledgements}

Not applicable.

\section{Funding}

The present study was supported by the China Postdoctoral Science Foundation (grant no. 2016M592513), the Project of Administration of Traditional Chinese Medicine of Guangdong Province of China (grant no. 20181114), Guangzhou Science and Technology Project (grant no. 201508020050) and the Guangzhou Science and Technology Project (grant no. 201604020003).

\section{Availability of data and materials}

The datasets used and/or analyzed during the current study are available from the corresponding author on reasonable request.

\section{Authors' contributions}

$\mathrm{XZ}$ and MD conceived the study, designed the experiments, analyzed the data and prepared the manuscript. MD and MZ were major contributors in writing the manuscript. XZ, CL, $\mathrm{MD}$ and $\mathrm{MZ}$ selected the subject and obtained samples for the present study. XZ, MD and MZ performed the experiments. All authors have read and approved the manuscript.

\section{Ethics approval and consent to participate}

The Ethics Committee of the Guangdong Provincial Hospital of Chinese Medicine (Guangzhou, China) approved the study protocol and all participants provided written informed consent.

\section{Patient consent for publication}

All participants provided written informed consent for publication.

\section{Competing interests}

The authors declare no conflicts of interest.

\section{References}

1. Yang G, Wang Y, Zeng Y, Gao GF, Liang X, Zhou M, Wan X, Yu S, Jiang Y, Naghavi M, et al: Rapid health transition in China, 1990-2010: Findings from the global burden of disease study 2010. Lancet 381: 1987-2015, 2013.

2. Lozano R, Naghavi M, Foreman K, Lim S, Shibuya K, Aboyans V Abraham J, Adair T, Aggarwal R, Ahn SY, et al: Global and regional mortality from 235 causes of death for 20 age groups in 1990 and 2010: A systematic analysis for the global burden of disease study 2010. Lancet 380: 2095-2128, 2012. 
3. He Y, Li Y, Chen Y, Feng L and Nie Z: Homocysteine level and risk of different stroke types: A meta-analysis of prospective observational studies. Nutr Metab Cardiovasc Dis 24: 1158-1165, 2014.

4. Zhao M, Wang X, He M, Qin X, Tang G, Huo Y, Li J, Fu J, Huang $\mathrm{X}$, Cheng $\mathrm{X}$, et al: Homocysteine and stroke risk: Modifying effect of methylenetetrahydrofolate reductase C677T polymorphism and folic acid intervention. Stroke 48: 1183-1190, 2017.

5. Boldyrev AA: Molecular mechanisms of homocysteine toxicity. Biochemistry (Mosc) 74: 589-598, 2009.

6. Wallin A, Sjögren M, Edman A, Blennow K and Regland B: Symptoms, vascular risk factors and blood-brain barrier function in relation to CT white-matter changes in dementia. Eur Ncurol 44: 229-235, 2000.

7. Lominadze D, Roberts AM, Tyagi N, Moshal KS and Tyagi SC: Homocy-steine causes cerebrovascular leakage in mice. Am J Physiol Heart Circ Physiol 290: H1206-H1213, 2006.

8. Kamath AF, Chauhan AK, Kisucka J, Dole VS, Loscalzo J, Handy DE and Wagner DD: Elevated levels of homocysteine compromise blood-brain barrier integrity in mice. Blood 107: 591-593, 2006.

9. Lee H, Kim JM, Kim HJ, Lee I and Chang N: Folic acid supplementation can reduce the endothelial damage in rat brain microvasculature due to hyperhomocysteinemia. J Nutr 135 : 544-548, 2005

10. Tyagi SC, Lominadze D and Roberts AM: Homocysteine in microvascular endothelial cell barrier permeability. Cell Biochem Biophys 43: 37-44, 2005.

11. Mo J, Yang R, Li F, Zhang X, He B, Zhang Y, Chen P and Shen Z Scutellarin protects against vascular endothelial dysfunction and prevents atherosclerosis via antioxidation. Phytomedicine 42 : 66-74, 2018.

12. Yuan Y, Fang M, Wu CY and Ling EA: Scutellarin as a potential therapeutic agent for microglia-mediated neuroinflammation in cerebral ischemia. Neuromolecular Med 18: 264-273, 2016.

13. Du X, Chen C, Zhang M, Cai D, Sun J, Yang J, Hu N, Ma C, Zhang L, Zhang J and Yang W: Scutellarin reduces endothelium dysfunction through the PKG-I pathway. Evid Based Complement Alternat Med 2015: 430271, 2015.

14. Guo LL, Guan ZZ, Huang Y, Wang YL and Shi JS: The neurotoxicity of $\beta$-amyloid peptide toward rat brain is associated with enhanced oxidative stress, inflammation and apoptosis, all of which can be attenuated by scutellarin. Exp Toxicol Pathol 65: 579-584, 2013.

15. Conlan RS, Pisano S, Oliveira MI, Ferrari M and Mendes Pinto I Exosomes as reconfigurable therapeutic systems. Trends Mol Med 23: 636-650, 2017

16. Xin H, Li Y, Cui Y, Yang JJ, Zhang ZG and Chopp M: Systemic administration of exosomes released from mesenchymal stromal cells promote functional recovery and neurovascular plasticity after stroke in rats. J Cereb Blood Flow Metab 33: 1711-1715, 2013.

17. Janas AM, Sapoń K, Janas T, Stowell MH and Janas T: Exosomes and other extracellular vesicles in neural cells and neurodegenerative diseases. Biochim Biophys Acta 1858: $1139-1151,2016$

18. Lehotsky J, Petras M, Kovalska M, Tothova B, Drgova A and Kaplan P: Mechanisms involved in the ischemic tolerance in brain: Effect of the homocysteine. Cell Mol Neurobiol 35: 7-15, 2015.
19. Wu CY, Fang M, Karthikeyan A, Yuan Y and Ling EA: Scutellarin attenuates microglia-mediated neuroinflammation and promotes astrogliosis in cerebral ischemia-a therapeutic consideration. Curr Med Chem 24: 718-727, 2017.

20. Corrigan F, Mander KA, Leonard AV and Vink R: Neurogenic inflammation after traumatic brain injury and its potentiation of classical inflammation. J Neuroinflammation 13: 264, 2016.

21. Sun X, Chen RC, Yang ZH, Sun GB, Wang M, Ma XJ, Yang LJ and Sun XB: Taxifolin prevents diabetic cardiomyopathy in vivo and in vitro by inhibition of oxidative stress and cell apoptosis. Food Chem Toxicol 63: 221-232, 2014.

22. Yang J, Ju B, Yan Y, Xu H, Wu S, Zhu D, Cao D and Hu J: Neuroprotective effects of phenylethanoid glycosides in an in vitro model of Alzheimer's disease. Exp Ther Med 13: 2423-2428, 2017

23. Wang JS, Bojovic D, Chen Y and Lindgren CA: Homocysteine sensitizes the mouse neuromuscular junction to oxidative stress by nitric oxide. Neuroreport 29: 1030-1035, 2018.

24. Wang X, Cui L, Joseph J, Jiang B, Pimental D, Handy DE, Liao R and Loscalzoa J: Homocysteine induces cardiomyocyte dysfunction and apoptosis through p38 MAPK-mediated increase in oxidant stress. J Mol Cell Cardiol 52: 753-760, 2012.

25. Sydow K, Hornig B, Arakawa N, Bode-Böger SM, Tsikas D, Münzel T and Böger RH: Endothclial dysfunction in patients with peripheral artcrial disease and chronic hyperhomocys-teinemia:potential role of ADMA. Vasc Med 9: 93-101, 2004.

26. Stremersch S, Vandenbroucke RE, Van Wonterghem E, Hendrix A, De Smedt SC and Raemdonck K: Comparing exosome-like vesicles with liposomes for the functional cellular delivery of small RNAs. J Control Release 232: 51-61, 2016.

27. Zhang HG and Grizzle WE: Exosomes: A novel pathway of local and distant intercellular communication that facilitates the growth and metastasis of neoplastic lesions. Am J Pathol 184: $28-41,2014$.

28. Jiao H, Wang Z, Liu Y, Wang P and Xue Y: Specific role of tight junction proteins claudin-5, occludin, and ZO-1 of the blood-brain barrier in a focal cerebral ischemic insult. J Mol Neurosci 44: 130-139, 2011.

29. Li Y, Li Q, Pan CS, Yan L, Hu BH, Liu YY, Yang L, Huang P, Zhao SY, Wang CS, et al: Bushen huoxue attenuates diabetes-induced cognitive impairment by improvement of cerebral microcirculation: Involvement of RhoA/ROCK/moesin and src signaling pathways. Front Physiol 9: 527, 2018.

30. Furuse M, Hirase T, Itoh M, Nagafuchi A, Yonemura $S$ and Tsukita S: Occludin: A novel integral membrane protein localizing at tight junctions. J Cell Biol 123: 1777-1788, 1993.

31. Ohtsuki S, Sato S, Yamaguchi H, Kamoi M, Asashima T and Terasaki T: Exogenous expression of claudin-5 induces barrier properties in cultured rat brain capillary endothelial cells. J Cell Physiol 210: 81-86, 2007.

This work is licensed under a Creative Commons Attribution-NonCommercial-NoDerivatives 4.0 International (CC BY-NC-ND 4.0) License. 